\title{
Black hole mass estimations: limitations and uncertainties
}

\author{
Marianne Vestergaard ${ }^{* a, b, c}$, Kelly D. Denney ${ }^{b, d}$, Xiaohui Fan ${ }^{c}$, Jens Juel Jensen ${ }^{b}$, \\ Brandon C. Kelly ${ }^{e, f}$, Patrick S. Osmer ${ }^{g}$, Bradley M. Peterson ${ }^{g}$, Christina A. Tremonti ${ }^{h}$ \\ ${ }^{a}$ Freja Fellow; Marie Curie Fellow \\ ${ }^{b}$ Dark Cosmology Centre, Niels Bohr Institute, Juliane Maries Vej 30, DK-2100 Copenhagen Ø \\ ${ }^{c}$ Department of Astronomy and Steward Observatory, University of Arizona, 933 N. Cherry \\ Avenue, Tucson, AZ, USA \\ d DARK Postdoctoral Fellow \\ ${ }^{e}$ Hubble Postdoctoral Fellow \\ ${ }^{f}$ Harvard-Smithsonian Center for Astrophysics, 60 Garden Street, Cambridge, Boston, USA \\ ${ }^{g}$ Department of Astronomy, Ohio State University, Columbus, OH, USA \\ ${ }^{h}$ Department of Astronomy, University of Madison-Wisconsin, USA
}

E-mail: vester@dark-cosmology.dk

\begin{abstract}
Mass scaling relations are a powerful tool for estimating virial masses of the central supermassive black holes that power distant quasars and active galactic nuclei owing in part to the relative ease with which they can be applied to large catalogs of spectral data. There is a large suite of mass scaling relations available in the literature, based on the three main broad emission lines: $\mathrm{H} \beta$, Mg II, and C IV. We summarize some of the issues to be aware of when choosing and using these relations, including those of special interest to Narrow Line Seyfert 1 galaxy studies. In particular, we advocate using high-quality spectral data and multiple emission lines for the most accurate mass estimates. In the literature certain scaling relations, including that based on C IV, are claimed to be unreliable. We show results indicating that all of the three broad lines $(\mathrm{H} \beta$, $\mathrm{Mg}$ II, or C IV) exhibit issues suggesting that none of them are more accurate mass estimators than the others. Only by using as many broad lines as possible can we hope to even out the slight deviations introduced by the individual lines. Denney et al. (these proceedings) provide convincing evidence against the use of low-quality data for mass estimates. We present additional evidence, including extensive simulations, that low-quality data is most likely to bias our mass estimates which can be quite significant for narrow-lined sources like NLS1s. Finally, we present results based on Bayesian statistical analysis of the SDSS DR3 luminosity and mass function sample which shows that the peak of the estimated Eddington luminosity ratios $\left(L_{\mathrm{BOL}} / L_{\mathrm{Edd}}\right)$ distribution will be overestimated and the distribution width underestimated unless the intrinsic uncertainties in the mass estimates and survey incompleteness are accounted for.
\end{abstract}

Narrow-Line Seyfert 1 Galaxies and their place in the Universe

April 4-6, 2011

Milano, Italy

${ }^{*}$ Speaker. 


\section{Virial Mass Estimates}

The most robust method for obtaining the mass of the central supermassive black hole powering active galactic nuclei is that of reverberation mapping (RM). It utilizes the variability properties of the central engine to determine the distance of the broad-emission line gas from the region emitting the ionizing continuum photons by means of the light travel time, $\tau$. By combining this distance $(R=c \tau)$ with the velocity of this gas that varies, as measured from the line width in the variable (i.e., $r m s$ ) spectrum, the virial central mass can be determined. In these proceedings, Brad Peterson (2011) provides a detailed review of this method and the associated assumptions and caveats to which the interested reader is referred. The distance of the broad-line gas, $R$, scales with the nuclear continuum luminosity [e.g., Kaspi et al. 2005; Bentz et al. 2006, 2009; see also the contribution by Misty Bentz (2011) for the most recent updates]. This $R-L$ relationship allows us to obtain an estimate of the virial mass of the central black hole with relative ease using an individual (i.e., single-epoch) spectrum of the active nucleus from which the velocity, $v$, of the $\mathrm{H} \beta$ gas is approximated by the FWHM of $\mathrm{H} \beta$ and its distance $R$ is approximated by the power-law continuum luminosity $L\left(5100 \AA\right.$ ) (e.g., Kaspi et al. 2000; Vestergaard 2002): $M_{\mathrm{BH}}=f \times v^{2} \times R / G(f$ is the fudge-factor containing the unknown characteristics of the geometry, structure, and the details of the velocity field and its inclination along our line-of-sight; see Peterson 2011). This is a particularly powerful method for obtaining black hole mass estimates of distant quasars (e.g., Vestergaard 2004; McLure \& Dunlop 2004; Woo et al. 2004; Kollmeier et al. 2006; Vestergaard et al. 2008; Shen et al. 2008) by using Mg II (e.g., McLure \& Jarvis 2002; Vestergaard \& Osmer 2009) and C IV (Vestergaard 2002; Warner et al. 2003; Vestergaard \& Peterson 2006) line widths to probe the broad-line region velocities when the Balmer lines are redshifted out of our observing window.

These single-epoch mass estimates (or 'mass scaling relations') for active galactic nuclei are, afterall, approximations to the robust RM masses, yet it is intriguing just how well they perform, given their crude nature: accuracies are a factor of $\sim 4$ on an absolute scale and a factor of 2.5 -3 relative to the RM masses (e.g., Vestergaard \& Peterson 2006). One can contrast this to the complex modeling of stellar orbits based on high-spatial resolution imaging that is necessary to estimate the mass of the central black hole in quiescent galaxies (see e.g. review by Ferrarese \& Ford 2005). The power of these relatively simple scaling relations thus becomes clear.

While an accuracy of a factor of a few is good, we would like to do better - and we can do better. We see scatter in the single-epoch estimates relative to the RM masses and scatter of AGN black hole masses around their $M_{\mathrm{BH}}-\sigma_{*}$ relation. There is a good potential for reducing this scatter. As reported by Brad Peterson and Misty Bentz at this meeting (Peterson 2011; Bentz 2011) by careful modeling of the host galaxy light distribution and computing the contribution of this light distribution in each of the spectral apertures used during the monitoring campaigns, we now have an accurate account of the star-light contamination for all RM AGN with a robust black hole mass determination. As a result the scatter in the $R-L$ relation is reduced and the slope is consistent with 0.5 to within $1 \sigma$ as compared to the early relationship (Kaspi et al. 2005) where the host galaxy contamination was not taken into account. In addition, by using only the best monitoring data in which the delay in the emission line response to continuum variations are visibly obvious from the raw data, the scatter in the $R-L$ relation is reduced to as little as 0.11 dex! (Peterson 2010).

We are now aware of a few other issues that affect the mass estimates - in excess of the 
unknown BLR inclination - which we should be able to address now or in the near future. These include: (1) effects of radiation pressure on the BLR gas, if relevant (see e.g., Marconi 2011), (2) luminosity color-correction between the optical and UV regions (Denney et al. 2011), (3) narrowline contamination of specific importance for NLS1 galaxies (Denney et al. 2011), (4) uncertainties inflicted by source variability (Woo 2011), (5) effects of low S/N data resulting in systematic biases in the line measurements (Denney et al. 2011) and in increasing the uncertainties in the line width measurements in general, and (6) the current Mg II and C IV-based mass scaling relations are not appropriate for NLS1s. I will focus on the effects of low-S/N data and the mass scaling relations for NLS1s in the following.

\section{The Power of Multiple Emission Lines for Mass Estimates}

There are a couple of important issues to keep in mind when using scaling relations to determine black hole masses of AGNs and quasars. At present there is a large suite of scaling relations present in the literature (e.g., McLure \& Jarvis 2002; Vestergaard 2002; McLure \& Dunlop 2004; Warner et al. 2003; Wu et al. 2004; Vestergaard \& Peterson 2006; McGill et al. 2008; Vestergaard \& Osmer 2009; Wang et al. 2009). For the same broad emission line (width) there are different relations and some works advocate using the line luminosity as oppose to the continuum luminosity to estimate the size of the BLR region. Also, some of the relations are based on outdated versions of the $R-L$ relation and/or not the most updated analysis of the reverberation mapping data. Hence, it can be a confusing task to select the appropriate scaling relationship. My advice is summarized in the following.

\section{Comparing mass estimates based on different lines?}

If you want to compare mass estimates based on two different emission lines, do make sure that the scaling relations for these emission line widths are in fact on the same mass scale. If not, one will naturally bias one's study as this will introduce an offset that has no basis in reality. Dietrich \& Hamann (2004) compared mass estimates based on H $\beta$ (or C IV) and $\mathrm{Mg}$ II scaling relations from two different studies and found a difference of up to a factor of five! Therefore, the mass scaling relations should be selected with care.

\section{Do you have more than one emission line?}

If you have more than one of the three emission lines for which a mass scaling relation exists then you are highly recommended to use all applicable lines to constrain the black hole mass. Some authors restrict their analysis to a single emission line in which they appear to have greater faith. This is not recommended for a few reasons. For one, I will argue later that all emission lines have their own issues and no single emission line is perfect for mass estimates. Second, the RM masses were determined by making use of multiple emission lines to determine the mass. As shown in Figure 1, all the broad emission lines (with exception of Lyman- $\alpha$ and $\mathrm{Mg}$ II which were not monitored) exhibit a virial relationship in concert (more such virial relationships are presented by Peterson \& Wandel 2000 and in Fig. 1 of Peterson 2011). Furthermore, the measurements slide up and down along the virial relationship as the source brightens and weakens, as one would expect if the BLR velocities are governed by 


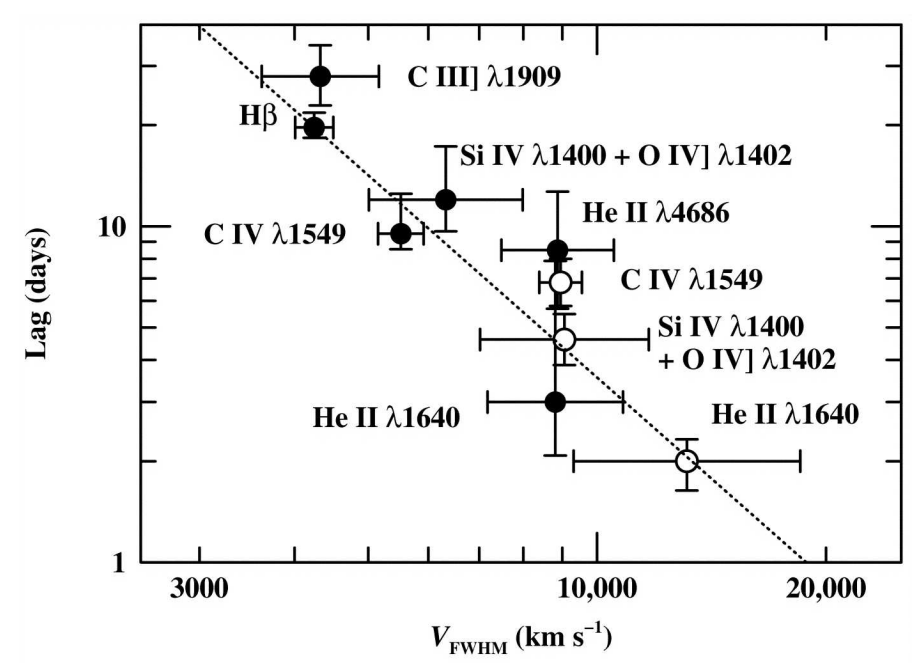

Figure 1: Virial relationship for Seyfert 1 galaxy, NGC 5548: the measured time delay (lag) in days for individual lines are shown as a function of the velocity of the line-emitting gas in $\mathrm{km} \mathrm{s}^{-1}$ at two different epochs (open and solid symbols, respectively; open circles: 1993, solid circles: 1989). The individual emission lines monitored are marked in the diagram. The dotted line shows this inverse relationship for a virial mass of $6.8 \times 10^{7} M_{\odot}$. This diagram emphasizes that (a) it is the collective power of all the monitored emission lines that yields the virial mass, (b) some scatter around the relation is expected for individual lines, (c) the emission lines 'slide' up and down this relation with varying continuum luminosity, and (d) the highionization lines are emitted closer to the central source and have - as anticipated - a larger velocity than the lower ionization lines. [Figure 1 of Peterson \& Wandel (1999)]. Figure is courtesy of Bradley M. Peterson.

the black hole gravity. However, the scatter in this virial relationship illustrates that a single emission line will not give an accurate measurement of the black hole mass. It is only by using all the emission lines that we can hope to even out the uncertainties associated with each individual line. This is similar to attempting to establish a linear relationship with less than three data points: at best you can only get a crude estimate of the slope or the amplitude (in the case of a single data point). Having three or more data points increases the probability of establishing the intrinsic relationship.

To our knowledge there is, at present, only one set of scaling relationships for which all three lines (H $\beta$, and especially Mg II and C IV) are on the same mass scale, namely those presented by Vestergaard \& Peterson (2006) and Vestergaard \& Osmer (2009). In addition, these relations are calibrated to the most recent updated analysis of the reverberation mapping sample (Peterson et al. 2004).

It is worth noting that for the $\mathrm{H} \beta$ and $\mathrm{Mg}$ II relationships in these studies, the line widths entering these relations are those of the broad emission line component only (as this has been misinterpreted; e.g., Shen et al. 2011). That is, the narrow line component contributing to the line profile is modeled and subtracted before the line width is measured. This is needed since the narrow-line gas velocities reflect the gravity of the enclosed mass at the distance of the Narrow Line Emitting Region (NLR), and therefore reflect not just the mass of the black hole. This narrow line component model is based on the velocity dispersion of the 
NLR as estimated from the widths of the [OIII] 5007 and [OII] 3727 emission lines. A NLR contribution to the C IV line is at most rather weak (or absent) (Wills et al., 1993). Therefore, there is no need for a correction for a narrow-line component in the $\mathrm{C}$ IV line.

\section{Discard bad data!}

It is now more clear than ever (see e.g., Denney et al. 2009, 2011) that data suffering from low $\mathrm{S} / \mathrm{N}$ and/or strong absorption in the emission line will bias the mass estimates if used. It has been common to model profiles of low $\mathrm{S} / \mathrm{N}$ with smooth functions to avoid noise-spikes to skew the measurements, but this does not in fact alleviate the problem as the low-S/N profile will systematically bias the estimates of the average velocity field (see Denney et al. 2009 for details). Also, it is not recommended that one attempts to 'correct' for absorption in the peak of the profile (or strong absorption in the wing) as any such correction will be no more than a random guess since there are rather limited constraints on the absorption in the data themselves. Such data should simply be discarded for the purpose of black hole mass estimates. Any line width measurements of such line profiles will always be highly unreliable.

\section{The C IV Line in NLS1s}

We have known for a while that the C IV profile of NLS1s tend to exhibit a blue asymmetric (triangular) shape, suggestive that these systems may have strong high-ionization outflows. In particular, Leighly (2000) found that the tendency of the C IV blue asymmetry increases for the high luminosity NLS1s. This asymmetry may be related to the high accretion rates thought to characterize the NLS1s. However, since in this case the C IV line profile appears to have a significant contribution from gas with non-Keplerian (or non-gravitationally induced) velocities, the C IV-based mass estimates are not likely representative of the intrinsic black hole mass (or, for that matter, $\mathrm{H} \beta$-based masses). Therefore, the current $\mathrm{C}$ IV-based scaling relations should not be applied to NLS1s (see also Vestergaard 2004 for a discussion of this issue for NLS1s).

\section{The C IV Line in Quasars - Are They Problematic?}

Quasars are also expected to be highly accreting objects, although to a less extreme degree than NLS1s given their larger central masses. Thus, a natural concern is whether the C IV profile of luminous high- $z$ quasars is representative of the virialized BLR that we expect from the RM results obtained in the local universe? While quasars do not have C IV profiles that resemble those of NLS1s (e.g., Vestergaard 2004) it is well-known that some quasars have C IV profiles that are blueshifted relative to the systemic redshift (e.g., Wilkes 1984). Richards et al. (2002) studied a few thousand quasars in the Sloan Digital Sky Survey (SDSS) with such blueshifts and found that the blueshift correlated with a decreasing equivalent width (the Baldwin effect) and a slight increase in line width. However, their analysis of composite spectra of quasars with a range of C IV blueshifts showed that the average broadening in the most blueshifted bin amounted to an increase of only $15 \%$ in the FWHM of the line which corresponds to an increase of about $30 \%$ in the mass values. At present, this is well within the uncertainties in the mass estimates. However, further 


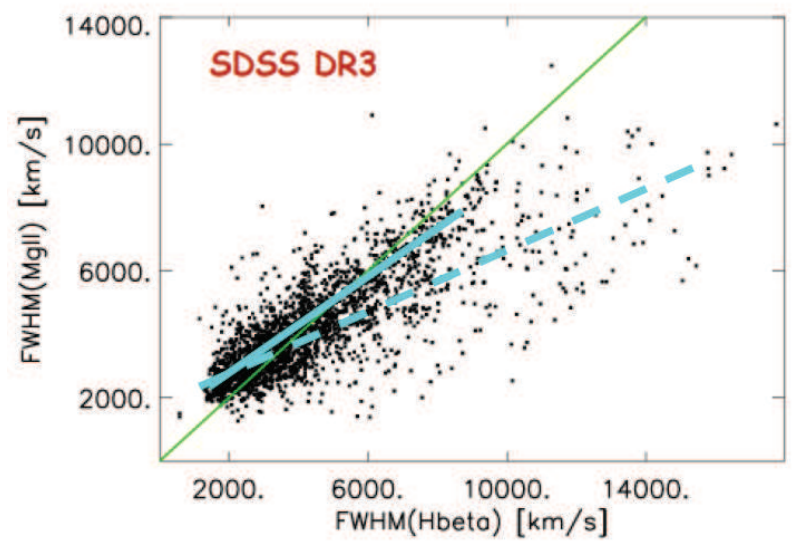

Figure 2: Relationship between the FWHM of the $\mathrm{Mg}$ II and $\mathrm{H} \beta$ emission lines for objects in the SDSS DR3 quasar catalog with spectral data of $\mathrm{S} / \mathrm{N} \geq 5$. The thin (green) line indicates a one-to-one relation. The solid blue line indicates the tilt of the high-density distribution of the data points. The dashed blue line shows the tilt of the relation for the broadest $\mathrm{H} \beta$ lines. This distribution of line widths shows that $\mathrm{FWHM}(\mathrm{Mg}$ II) is not always a good proxy of the $\mathrm{H} \beta$ FWHM. In particular, for line widths lower than about $3000 \mathrm{~km} \mathrm{~s}^{-1}$ the $\mathrm{Mg}$ II line overestimates the width of $\mathrm{H} \beta$. A consequence is that the mass scaling relation for $\mathrm{Mg}$ II is not strictly defined for NLS1s, and care must be exercised in use of Mg II scaling relations for NLS1s in general (the uncertainties in the mass estimates are clearly larger).

investigation of this issue is, of course, needed. As our uncertainties in the mass estimates improve further, this systematic change in line width needs to be taken into account.

However, it is worth keeping in mind that for the objects for which we have been able to perform the relevant test (see e.g., Fig. 1 of Peterson 2011), we see the C IV line being virialized similarly to the other optical and UV broad emission lines that have been monitored (Si IV] $+\mathrm{O}$ IV] $\lambda$ 1400, C IV $\lambda$ 1549, He II $\lambda$ 1640, C III] $\lambda$ 1909, He II $\lambda$ 4686, H $\beta$; Peterson $\&$ Wandel 1999 , 2000; Figure 1). The fact that the size of the C IV emitting region of a redshift 2 quasar is found to lie right on the extension of the local C IV broad-line region-size to luminosity relation (Kaspi et al. 2007) also suggests that the broad-line regions of distant luminous quasars is structured and behave like nearby AGNs. This may also be manifested in the fact that quasar spectra appear very similar across broad redshift ranges (at least out to redshifts of 4.75 where the data can be directly compared) and for a range of luminosities (Dietrich et al. 2002).

\section{No Broad Emission Line is Perfect}

Contrary to what is commonly reported in the literature, the $\mathrm{Mg}$ II line widths do not exhibit a tight one-to-one relationship with the $\mathrm{H} \beta$ line width. In Figure 2 we compare $\mathrm{Mg}$ II and $\mathrm{H} \beta$ line widths of several thousand SDSS DR3 quasars measured only for spectral data with median $\mathrm{S} / \mathrm{N} \geq 5$. There is clearly some scatter in the relationship which is slightly tilted relative to a oneto-one relation and has a long tail toward rather broad $\mathrm{H} \beta$ profiles. Some of these very broad $\mathrm{H} \beta$ profiles are double-peaked, suggesting that perhaps in these cases, the $\mathrm{H} \beta$ profile may either be a poor measure of the BLR velocity field, or the Mg II profile is (since it is clearly too narrow in 


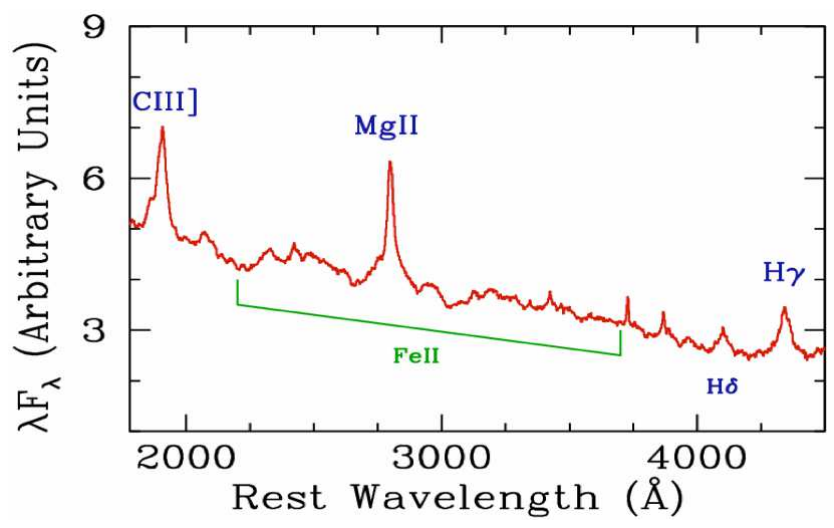

Figure 3: The spectral region around $\mathrm{Mg}$ II $\lambda 2800$ is clustered with thousands of broad Fe II emission lines that blend with themselves and with $\mathrm{Mg}$ II. About half of the Mg II line flux is buried in the Fe II emission. (Figure is based on data from study by Francis, Hooper, \& Impey 1993).

comparison with the $\mathrm{H} \beta$ profile). An added complication is the location of $\mathrm{Mg}$ II in the middle of the small blue bump of thousands of iron emission lines that blend with themselves and with $\mathrm{Mg}$ II (Vestergaard \& Wilkes 2001): establishing accurately the location of the underlying continuum and the full profile shape of $\mathrm{Mg}$ II is prone to errors and systematics since half the $\mathrm{Mg}$ II profile is submerged in the Fe II emission (Fig. 3).

For redshifts between 1.5 and 2.2 both Mg II and C IV appear in the SDSS observing window. In Figure 4 the distribution of C IV and Mg II line widths are shown for the over 5000 quasar spectra with median $\mathrm{S} / \mathrm{N}$ above 5 in this redshift range. There is significant scatter and deviations from a linear relationship between the two line widths. This is also seen by Shen et al. (2008); some

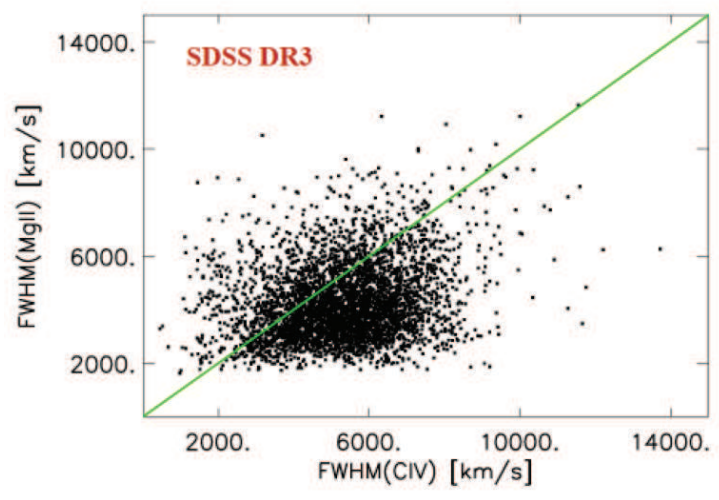

Figure 4: Relationship between FWHM(Mg II) and FWHM(C IV) for objects in the SDSS DR3 quasar catalog with spectral data with median $\mathrm{S} / \mathrm{N}$ level $\geq 5$. The thin (green) line indicates a one-to-one relation. The distribution of data points shows that the $\mathrm{Mg}$ II line is often narrower than the $\mathrm{C}$ IV emission line, a tendency also seen for Mg II when compared to $\mathrm{H} \beta$ (Figure 2). The cause is currently unknown, but does emphasize that all three emission lines of $\mathrm{H} \beta, \mathrm{Mg}$ II, and C IV may have issues. That is one reason that the use of multiple emission lines are advocated to obtain a better measure of the intrinsic black hole mass. 
of the scatter appears to be related to the blueshift sometimes observed for the C IV line profiles: the stronger the blueshift, the more deviant are the mass estimates based on $\mathrm{Mg}$ II relative to those based on C IV. More work is needed to investigate the cause of this deviation as well as the potential for generating corrections to either the $\mathrm{Mg}$ II or the $\mathrm{C}$ IV line width.

However, it is worth noting that although C IV is often blamed for these offsets (and thereby for being a less reliable mass estimator) the $\mathrm{Mg}$ II line width has been reported to depend on the source Eddington ratio, $L_{\mathrm{BOL}} / L_{\mathrm{Edd}}($ Onken $\&$ Kollmeier 2008). Furthermore, there appears to be a systematic narrowing of the Mg II line with redshift for $z \gtrsim 1.4$ - the very redshift above which the Mg II and C IV lines appear in the same SDSS spectrum and thus can be compared directly.

Based on this observational evidence, it is not at all clear that C IV is the problematic emission line for mass estimates. There are clearly issues with each of the three lines, $\mathrm{H} \beta, \mathrm{Mg}$ II and $\mathrm{C}$ IV, that suggest that there are variations in the velocity fields and/or the conditions in the broad emission line region that prevent these line widths from exhibiting linear and tight correlations (see e.g., Baldwin et al. 1995). Perhaps this is not too surprising, considering that the broad-line region must necessarily be a dynamic and non-static region - whether it consists of orbiting 'clouds' or outflowing winds (e.g., Murray et al. 1995; Elvis 2000).

\section{A Word of Caution: Mg II Scaling Relation is Not Valid for NLS1s}

The fact that the FWHM measurements of the high-S/N SDSS data show a systematic deviation from a one-to-one relationship between FWHM(Mg II $)$ and $\mathrm{FWHM}(\mathrm{H} \beta)$ for FWHM less than $\sim 2500-3000 \mathrm{~km} \mathrm{~s}^{-1}$ means that this scaling relation is not valid for NLS1s (Vestergaard \& Osmer 2009). The Mg II line widths overestimate the $\mathrm{H} \beta$ line widths and hence the black hole mass in this case. The effect is clearly larger for more narrow $\mathrm{H} \beta$ lines. Further investigation is needed to clarify the cause of this deviation and find a way to correct for this offset, if possible.

\section{The S/N Ratio of the Data Does Matter!}

Denney et al. (2011) have already provided some convincing arguments that low $\mathrm{S} / \mathrm{N}$ data can skew our line width measurements also in a systematic manner. Since the line width enters the mass scaling relations to the second power, systematic differences (or larger scatter) in the measurements will propagate to significant differences in the mass values. In the following I provide some slightly different evidence that also disfavor the use of low $\mathrm{S} / \mathrm{N}$ data. Since large surveys typically produce many spectra of relatively low $\mathrm{S} / \mathrm{N}$ (owing to the way such surveys must necessarily be optimized), this is not a trivial point. With a large body of data (including low $\mathrm{S} / \mathrm{N}$ data) readily available, it is easy and very tempting to simply measure and analyze all the data regardless of its quality. However, the uncertainties associated with the low-S/N data are much larger than our mass scaling relations imply since the latter were obtained based on relatively high quality data.

Vestergaard et al. (2008) analyzed a subset of the DR3 quasar catalog in order to study the black hole mass functions at a range of redshifts. The analysis included a spectral decomposition of the quasar spectra into the contributions from a nuclear power-law continuum, a host galaxy contribution (for redshifts below 0.5 where the host contribution can be constrained), an Fe II emission spectrum, a Balmer continuum, plus broad and narrow emission line profiles. The models were 

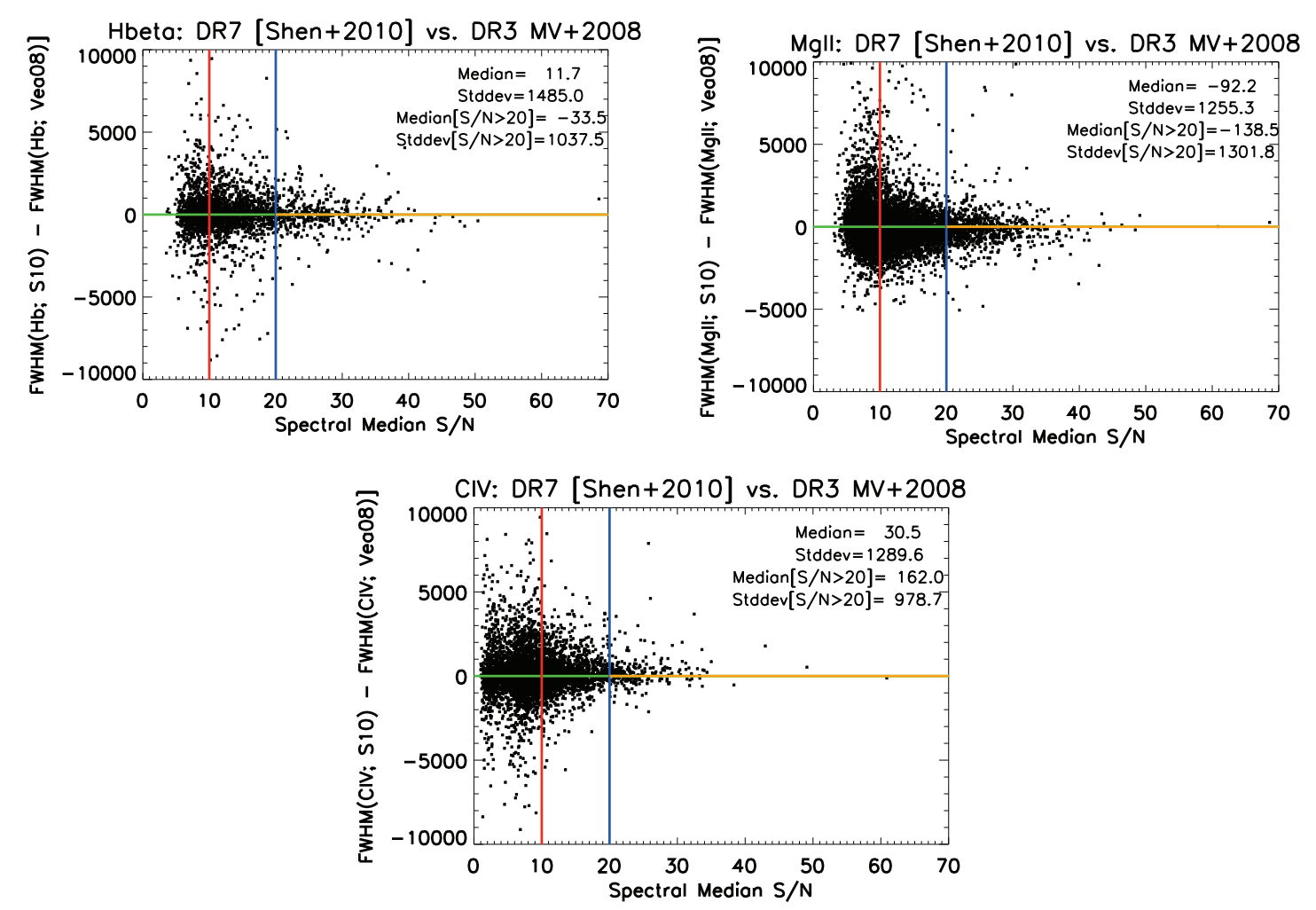

Figure 5: Measured line width differences shown as a function of spectral $\mathrm{S} / \mathrm{N}$ ratio for the data common between the studies of Shen et al. (2011) and Vestergaard et al. (2008). The panels show (top to bottom) the differences for the three emission lines $\mathrm{H} \beta, \mathrm{Mg}$ II, and C IV. These diagrams show that two different ways of measuring the same data typically yields different FWHM measurements. The differences increase with decreasing median spectral S/N. The median (and 1 standard deviation) FWHM differences are listed for the entire sample and for the subset having $\mathrm{S} / \mathrm{N}>20$. While these diagrams also show that differences must exist in the way the two studies measure the data, they are a stark reminder that measuring line widths in spectral data is not a trivial task (but involves uncertainties and perhaps systematics depending on the method; see also Denney et al. 2009) and low-S/N data is (at present) the worst enemy of accurate mass estimates.

optimized through a least-squares minimization routine. The FWHM measurements of the CIV, $\mathrm{Mg}$ II, and $\mathrm{H} \beta$ line profiles are directly compared to the measurements performed by Shen et al. (2011) on the exact same data in Figure 5. The differences in FWHM line widths are plotted as a function of the median $\mathrm{S} / \mathrm{N}$ ratio in the spectral data. These diagrams show that the deviations are clearly a function of the S/N level: the scatter and mean deviation increases with decreasing S/N. It also shows that two different methods of measuring line parameters can have a strong effect on the measurements - even at high $\mathrm{S} / \mathrm{N}$. It is noteworthy that none of the three emission lines perform better than the others. Therefore, one must be careful to use high $\mathrm{S} / \mathrm{N}$ data for the measurements if one needs more than an order of magnitude estimate of the black hole masses.

Needless to say, the precise method with which we actually perform the line measurements also matters (see the scatter for the high S/N data in Fig. 5 and the study by Denney et al. 2009). The studies of Rafiee \& Hall (2011a,b) also illustrate the important issue and potential implications 


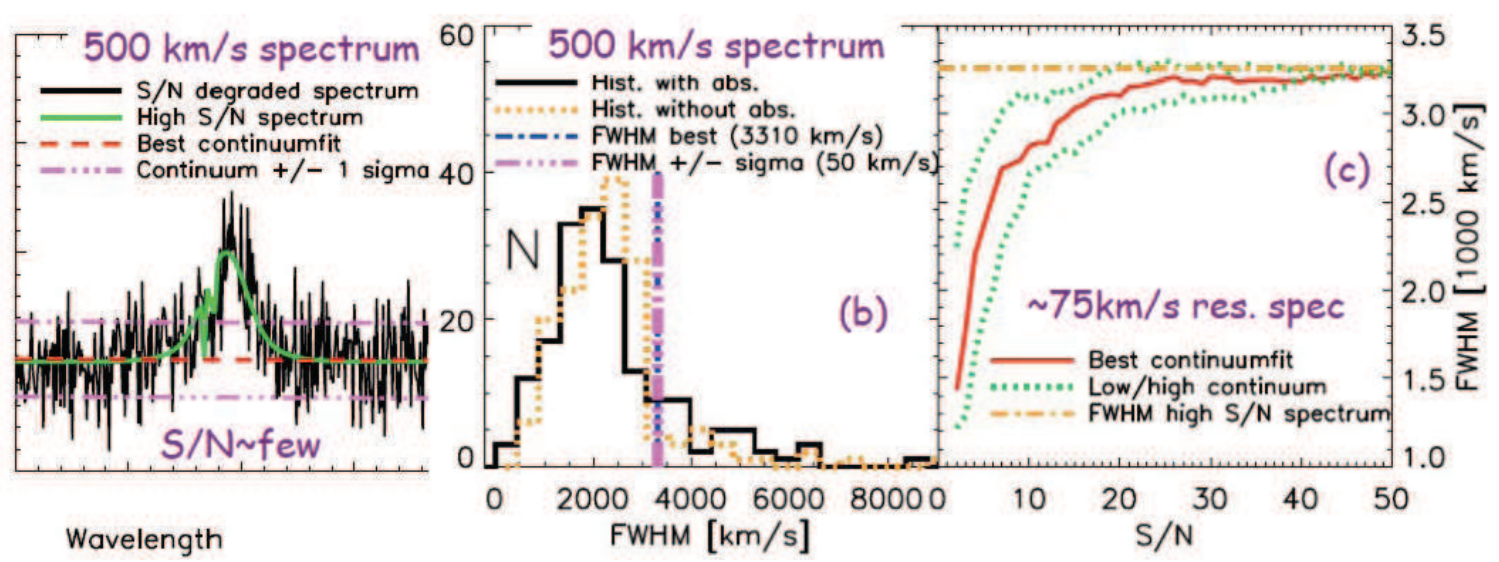

Figure 6: Simulations showing the implications of low $\mathrm{S} / \mathrm{N}$ data on our ability to measure or reconstruct the intrinsic (emitted) line width. A model spectrum is $\mathrm{S} / \mathrm{N}$ degraded before the line profile is measured. Left panel: the appearance of an emission line (with intrinsic width of $\mathrm{FWHM}=3310 \pm 50 \mathrm{~km} \mathrm{~s}^{-1}-$ measured on a profile with $\mathrm{S} / \mathrm{N}=50$ ) at a spectral resolution of $500 \mathrm{~km} \mathrm{~s}^{-1}$ and $\mathrm{S} / \mathrm{N} \sim 2-3$ (black curve). Superimposed on the line are two narrow absorption lines, each of widths of $\sim 300 \mathrm{~km} \mathrm{~s}^{-1}$. The emitted profile (with absorption) is shown by the green curve. Overplotted is the best continuum fit (red dashed) to the $\mathrm{S} / \mathrm{N}$ degraded spectrum (black curve) along with its $1 \sigma$ uncertainties (purple dot-dashed). The middle panel: the distribution of 250 FWHM measurements of this $\mathrm{S} / \mathrm{N}$ degraded spectrum (with and without the absorption features overlaid) is compared to the measurement made on the $\mathrm{S} / \mathrm{N}=50$ spectrum (vertical blue and purple dot-dashed lines). Right panel: Accuracy of FWHM measurements made on a model line with absorption as a function of $\mathrm{S} / \mathrm{N}$ level. In this case the simulations show the results of data obtained with a spectral resolution of $75 \mathrm{~km} \mathrm{~s}^{-1}$. This shows that high spectral resolution and high-S/N ( $220 /$ pixel) are needed to avoid a significant bias in the FWHM measurements. These are preliminary results of an extended ongoing investigation.

of how the line profiles are measured. We are in the process of investigating robust ways to measure the line profiles.

\section{Simulations: Narrow Lines and Low $\mathrm{S} / \mathrm{N}$}

Two of us (Jensen \& Vestergaard) are in the process of simulating the effects of low $\mathrm{S} / \mathrm{N}$ on relatively narrow emission lines, relevant for NLS1 galaxies, with different characteristics ${ }^{1}$ on our ability to measure the intrinsic line width (i.e., that emitted by the source) in data obtained with spectrographs of different spectral resolutions. The first results show, similar to Denney et al. (2009; also, Denney et al. 2011) a tendency (and larger probability) in low S/N data for significantly underestimating the line widths. This is enhanced if there is even weak intervening absorption superposed on the emission line (Figure 6b). As is seen in Fig. 6b, there is a large probability that we can underestimate the line width by $\sim 1000 \mathrm{~km} \mathrm{~s}^{-1}$. It is easier to detect and discern absorption lines in higher resolution spectra. These simulations show that a spectrum with S/N of 20-25 per pixel is needed to avoid a systematic underestimate of the line widths (Fig. 6c). These results are

\footnotetext{
${ }^{1}$ That is, the width, height, and shape of the emission line, plus the presence or absence of absorption lines, and the width and specific location(s) of absorption lines relative to the emission line peak.
} 


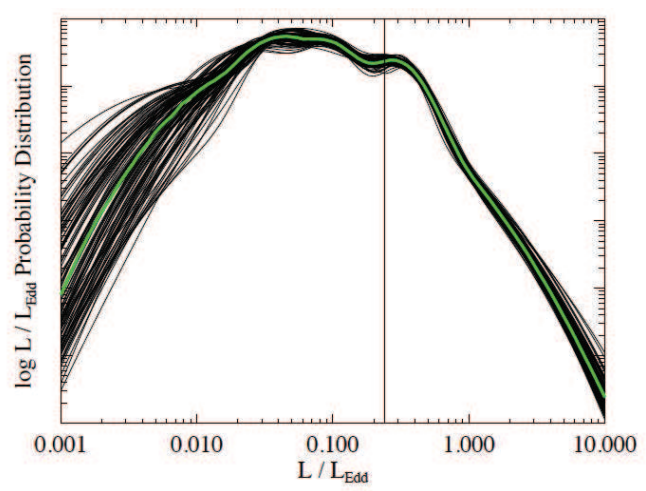

Figure 7: Probability distribution of the Eddington luminosity ratio $L_{\mathrm{BOL}} / L_{\mathrm{Edd}}$ for quasars in the SDSS DR3 quasar luminosity (and mass) function sample. The black thin curves show 100 draws from the probability distribution function. The thick green curve shows the mean distribution. The $10 \%$ completeness limit at $z=1$ is shown by the vertical line. The kink at $L_{\mathrm{BOL}} / L_{\mathrm{Edd}} \sim 0.3$ is not real, but an artifact of the assumption that the distribution can be reproduced by a mixture of log-normal functions. The distribution peaks at $L_{\mathrm{BOL}} / L_{\mathrm{Edd}} \sim 0.05$, but the exact value should be interpreted with caution as it falls below the $10 \%$ completeness limit. Nonetheless, this estimated distribution does suggest that most supermassive black holes in broad-lined quasars are not radiating near their Eddington limit (Figure 11 of Kelly et al. 2010).

preliminary in that all the measurements are performed directly on the simulated data and not on model fits to the noise-laden data. We do expect - as emphasized by Denney et al. (2009) - that model fits to the low-S/N data will not allow an unbiased reconstruction of the emitted line profile.

\section{Distribution of Eddington Luminosity Ratio}

One of the interpretations of NLS1s is that they contain relatively smaller central black hole masses that radiate at or near the Eddington limit. There is even some discussion that they may radiate above the limit (e.g., Collin \& Kawaguchi 2004). Broad-line quasars are also luminous sources and are often considered to be radiating at relatively high $L_{\mathrm{BOL}} / L_{\mathrm{Edd}} \lesssim 1$. This was emphasized by recent studies of the SDSS (McLure \& Dunlop 2004) and the AGES (Kollmeier et al. 2006) surveys of broad lined quasars which argue that the distribution of Eddington luminosity ratios, $L_{\mathrm{BOL}} / L_{\mathrm{Edd}}$, is rather narrow and centered at a mean $L_{\mathrm{BOL}} / L_{\mathrm{Edd}}$ value of $\sim 0.25$. The reality of this was questioned by Shen et al. (2008) who argue that the narrow distribution is due to a Malmquist type bias caused by the limited luminosity range. We have performed Bayesian statistical analysis of the DR3 quasar luminosity and mass function sample (Richards et al. 2006, Vestergaard et al. 2008) where survey incompleteness, the intrinsic uncertainties in the mass estimates, and the spectral measurement uncertainties are self-consistently taken into account (Kelly et al. 2010). The resulting $L_{\mathrm{BOL}} / L_{\mathrm{Edd}}$ distribution is shown in Figure 7 . The distribution is broader $(0.4 \mathrm{dex})$ and peaks at a lower value $(\sim 0.05)$ than reported earlier. Our results suggest that the previous works see a narrow $L_{\mathrm{BOL}} / L_{\mathrm{Edd}}$ distribution peaking at $\sim 0.25$ due to uncorrected incompleteness. These results are fully consistent with more recent and deeper studies (Gavignaud et al. 2008; Trump et al. 2009) and are also consistent with the analysis of Shen et al. (2008). The reader is referred to Kelly et al. (2010) for further details. The implications are that most supermassive black holes in 
type 1 quasars are not radiating near their Eddington limit. This result was obtained for all broadlined quasars and is perhaps not directly applicable to NLS1s. However, it does indicate that the available data may give us a misleading impression of these luminous sources if care is not exercised during our analysis and interpretation of the data. Collin \& Huré (2001) have additional and specific suggestions on the physical differences between broad-line Seyferts and NLS1s. Notably, Marconi et al. (2011) advocate that radiation pressure is relatively more significant for NLS1s.

\section{Summary}

The main points of this contribution can be summarized as follows:

- There is a large number of mass scaling relations in the literature from which to estimate the central black hole mass. However, not all equations are inter-calibrated to be on the same mass scale, so caution must be exercised. This is particularly important if you need to compare mass estimates based on one emission line (like Mg II) with that of another (like C IV). Otherwise one should expect to see offsets in mass estimates as has also been reported and has sometimes been attributed to problems with the mass estimation method.

- If you have multiple broad emission lines in your spectrum, use them all for a final mass estimate: multiple data points are better than one. The exception is, of course, if the line profiles are strongly absorbed, incomplete (truncated by the spectrograph), or the $\mathrm{S} / \mathrm{N}$ is low (see also Denney et al. 2011).

- Keep in mind that it does matter how you measure the line profile widths and what quality data you measure as you can otherwise systematically bias your measurements and thus your mass estimates (see also Denney et al. 2011 and Peterson 2011).

- Recall that the CIV emission line cannot be used for mass estimates in high-luminosity NLS1s as the profile may have significant contribution from gas with non-Keplerian motion.

- Beware that the current Mg II-based mass scaling relations are not particularly good for line widths below $\sim 2500 \mathrm{~km} \mathrm{~s}^{-1}$, at present (i.e., for NLS1s). There is not a one-to-one correspondence between the $\mathrm{H} \beta$ and Mg II line widths in this case: Mg II widths tend to overestimate the $\mathrm{H} \beta$ widths (and thus black hole masses).

- Bayesian analysis shows that the intrinsic distribution of quasar $L_{\mathrm{BOL}} / L_{\mathrm{Edd}}$ values is broader $\left(\sigma\left(L_{\mathrm{BOL}} / L_{\mathrm{Edd}}\right) \sim 0.4 \mathrm{dex}\right)$ and with a peak shifted toward lower values $\left(<L_{\mathrm{BOL}} / L_{\mathrm{Edd}}>\sim 0.05\right)$ than previously reported $\left(<L_{\mathrm{BOL}} / L_{\mathrm{Edd}}>\sim 0.25, \sigma\left(L_{\mathrm{BOL}} / L_{\mathrm{Edd}}\right) \sim 0.3 \mathrm{dex}\right)$. This implies that broad-lined quasars generally accrete at lower Eddington luminosity ratios than commonly assumed. The question is whether this also applies to NLS1s.

\section{Acknowledgments}

We are grateful for support of this research by NASA through grants HST-GO-10417 and HST-AR-12149 from the Space Telescope Science Institute. We wish to thank the organizers for a scientifically rewarding conference. 


\section{References}

[1] Baldwin, J., et al. 1995, Locally Optimally Emitting Clouds and the Origin of Quasar Emission Lines, ApJ, 455, L119

[2] Bentz, M. 2011, Black hole scaling relationships and NLS1s, In: Proceedings of the Workshop Narrow-Line Seyfert 1 Galaxies and Their Place in the Universe, PoS (NLS1) 033 (2011).

[3] Bentz, M.C., Peterson, B.M., Pogge, R.W., Vestergaard, M., Onken, C.A. 2006, The Radius-Luminosity Relationship for Active Galactic Nuclei: The Effect of Host-Galaxy Starlight on Luminosity Measurements, ApJ 644, 133

[4] Bentz, M.C., Peterson, B.M., Netzer, H., Pogge, R.W., Vestergaard, M. 2009, The Radius-Luminosity Relationship for Active Galactic Nuclei: The Effect of Host-Galaxy Starlight on Luminosity Measurements. II. The Full Sample of Reverberation-Mapped AGNs, ApJ 697, 160

[5] Collin, S., \& Huré, J.-M. 2001, Size-mass-luminosity relations in AGN and the role of the accretion disc, A\&A, 372, 50

[6] Collin, S., \& Kawaguchi, T. 2004, Super-Eddington accretion rates in Narrow Line Seyfert 1 galaxies., A\&A, 426, 797

[7] Denney, K.D., Peterson, B.M., Dietrich, M., Vestergaard, M., Bentz, M.C. 2009, Systematic Uncertainties in Black Hole Masses Determined from Single-Epoch Spectra, ApJ, 692, 246

[8] Denney, K. et al. 2011, Addressing systematic uncertainties in black hole mass measurements, In: Proceedings of the Workshop Narrow-Line Seyfert 1 Galaxies and Their Place in the Universe, POS (NLS1) 034 (2011).

[9] Dietrich, M. \& Hamann, F. 2004, Implications of Quasar Black Hole Masses at High Redshifts, ApJ, 611,761

[10] Dietrich, M. et al. al. 2002, Continuum and Emission-Line Strength Relations for a Large Active Galactic Nuclei Sample, ApJ 581, 912-924

[11] Elvis, M. 2000, A Structure for Quasars, ApJ, 545, 63

[12] Ferrarese, L. \& Ford, H. 2005, Supermassive Black Holes in Galactic Nuclei: Past, Present and Future Research, Space Science Reviews, Volume 116, Issue 3-4, pp. 523-624

[13] Francis, P.J., Hooper, E.J., Impey, C.D. 1993, The ultraviolet spectra of radio-loud and radio-quiet quasars, AJ, 106, 417

[14] Gavignaud, I., et al. 2008, Eddington ratios of faint AGN at intermediate redshift: evidence for a population of half-starved black holes, A\&A, 492, 637

[15] Kaspi, S., Smith, P.S., Netzer, H., Maoz, D., Jannuzi, B.T., Giveon, U. 2000, Reverberation Measurements for 17 Quasars and the Size-Mass-Luminosity Relations in Active Galactic Nuclei, ApJ, 533, 631

[16] Kaspi, S. et al. 2005, The Relationship between Luminosity and Broad-Line Region Size in Active Galactic Nuclei, ApJ, 629, 61

[17] Kaspi, S. et al. 2007, Reverberation Mapping of High-Luminosity Quasars: First Results, ApJ, 659, 997

[18] Kelly, B.C., et al. 2010, Constraints on Black Hole Growth, Quasar Lifetimes, and Eddington Ratio Distributions from the SDSS Broad-line Quasar Black Hole Mass Function, ApJ, 719, 1315 
[19] Kollmeier, J.A. et al. 2006, Black Hole Masses and Eddington Ratios at $0.3<z<4$, ApJ 648, 128

[20] Leighly, K. 2000, ASCA (and HST) observations of NLS1s, New Astronomy Reviews, Volume 44, Issue 7-9, p. 395-402

[21] Marconi, A. 2011, The effect of radiation pressure on virial black hole mass estimates: the case of narrow line Seyfert 1 galaxies, In: Proceedings of the Workshop Narrow-Line Seyfert 1 Galaxies and Their Place in the Universe, POS (NLS1) 040 (2011).

[22] McGill, K.L., Woo, J.-H., Treu, T., Malkan, M.M.A. 2008, Comparing and Calibrating Black Hole Mass Estimators for Distant Active Galactic Nuclei, ApJ, 673, 703

[23] McLure \& Dunlop 2004, The cosmological evolution of quasar black hole masses, MNRAS, 352 , 1390

[24] McLure \& Jarvis 2002, Measuring the black hole masses of high-redshift quasars, MNRAS, 337, 109

[25] Murray, N., Chiang, J., Grossman, S.A., Voit, G.M. 1995, Accretion Disk Winds from Active Galactic Nuclei, ApJ, 451, 498

[26] Onken, C.A. \& Kollmeier, J.A. 2008, An Improved Method for Using Mg II to Estimate Black Hole Masses in Active Galactic Nuclei, ApJ, 689, L13

[27] Peterson, B. M., 2010, Toward Precision Measurement of Central Black Hole Masses, In: Proceedings of the IAU Symposium 267: Co-Evolution of Central Black Holes and Galaxies, Vol. 267. p. 151-160

[28] Peterson, B. M., 2011, Masses of Black Holes in Active Galactic Nuclei: Implications for Narrow-Line Seyfert 1 Galaxies, In: Proceedings of the Workshop Narrow-Line Seyfert 1 Galaxies and Their Place in the Universe, PoS (NLS1) 032 (2011).

[29] Peterson, B.M. \& Wandel, A. 1999, Keplerian Motion of Broad-Line Region Gas as Evidence for Supermassive Black Holes in Active Galactic Nuclei, ApJ, 521, L95

[30] Peterson, B.M. \& Wandel, A. 2000, Evidence for Supermassive Black Holes in Active Galactic Nuclei from Emission-Line Reverberation, ApJ, 540, L13

[31] Peterson, B.M. et al. 2004, Central Masses and Broad-Line Region Sizes of Active Galactic Nuclei. II. A Homogeneous Analysis of a Large Reverberation-Mapping Database, ApJ, 613, 682

[32] Rafiee, A. \& Hall, P.B. 2011a, Supermassive Black Hole Mass Estimates Using Sloan Digital Sky Survey Quasar Spectra at $0.7<z<2$, ApJS, 194, 42

[33] Rafiee, A. \& Hall, P.B. 2011b, Biases in the quasar mass-luminosity plane, MNRAS, in press (arXiv: 1011.1268; DOI: 10.1111/j.1365-2966.2011.18910.x)

[34] Richards, G.T. et al. 2002, Broad Emission-Line Shifts in Quasars: An Orientation Measure for Radio-Quiet Quasars?, AJ, 124, 1

[35] Richards, G.T. et al. 2006, The Sloan Digital Sky Survey Quasar Survey: Quasar Luminosity Function from Data Release 3, AJ, 131, 2766

[36] Shen, Y. et al. 2008, Biases in Virial Black Hole Masses: An SDSS Perspective, ApJ, 680, 169

[37] Shen, Y. et al. 2011, A Catalog of Quasar Properties from Sloan Digital Sky Survey Data Release 7 , ApJS, 194, 45

[38] Trump, J.R. et al. 2009, Observational Limits on Type 1 AGN Accretion Rate in COSMOS, ApJ, 700, 49 
[39] Vestergaard, M. 2002, Determining Central Black Hole Masses in Distant Active Galaxies, ApJ, 571, 733

[40] Vestergaard, M. 2004, Early Growth and Efficient Accretion of Massive Black Holes at High Redshift, ApJ, 601, 676

[41] Vestergaard, M. \& Osmer, P.S. 2009, Mass Functions of the Active Black Holes in Distant Quasars from the Large Bright Quasar Survey, the Bright Quasar Survey, and the Color-selected Sample of the SDSS Fall Equatorial Stripe, ApJ, 699, 800

[42] Vestergaard, M. \& Peterson, B.M. 2006, Determining Central Black Hole Masses in Distant Active Galaxies and Quasars. II. Improved Optical and UV Scaling Relationships, ApJ, 641, 689

[43] Vestergaard, M. \& Wilkes, B.J. 2001, An Empirical Ultraviolet Template for Iron Emission in Quasars as Derived from I Zwicky 1, ApJS, 134, 1

[44] Vestergaard, M. et al. 2008, Mass Functions of the Active Black Holes in Distant Quasars from the Sloan Digital Sky Survey Data Release 3, ApJ, 674, L1

[45] Wang, J.-G., et al. 2009, Estimating Black Hole Masses in Active Galactic Nuclei Using the Mg II $\lambda 2800$ Emission Line, ApJ, 707, 1334

[46] Warner, C., Hamann, F., Dietrich, M. 2003, A Relation between Supermassive Black Hole Mass and Quasar Metallicity?, ApJ, 596, 72-84

[47] Wilkes, B.J. 1984, Studies of broad emission line profiles in QSO. I - Observed, high-resolution profiles, MNRAS, 207, 73

[48] Wills, B.J. et al. 1993, The narrow-line region of high-luminosity active galactic nuclei, ApJ, 410, 534

[49] Woo, J.-H. 2011, The overall uncertainty of single-epoch virial black hole mass estimators and its implication to the M-sigma relation, In: Proceedings of the Workshop Narrow-Line Seyfert 1 Galaxies and Their Place in the Universe, POS (NLS1) 039 (2011).

[50] Woo, J.-H., et al. 2004, The Fundamental Plane Evolution of Active Galactic Nucleus Host Galaxies, ApJ, 617, 903

[51] Wu, X.-B., Wang, R., Kong, M.Z., Liu, F.K., Han, J.L. 2004, Black hole mass estimation using a relation between the BLR size and emission line luminosity of $A G N, \mathrm{~A} \& \mathrm{~A}, 424,793$ 